\title{
Analysis, Modeling, and Compensation of Friction for Scaled Bilateral Control
}

\author{
Yosuke Mizutani* $^{*}$ Student Member, Seiichiro Katsura* Senior Member
}

(Manuscript received April 24, 2013, revised Jan. 18, 2014)

\begin{abstract}
At present, many kinds of robots are being developed for medical care. In medical care, operations often need to be performed at the micro-scale. This study was focused on a macro-micro bilateral control system that can transmit haptic sensations of a micro-environment between master and slave systems. The macro-micro bilateral control system amplifies disturbances; therefore, analysis, modeling and compensation of the disturbances were considered.
\end{abstract}

Keywords: macro-micro bilateral control system, viscous friction, coulomb friction, scaling

\section{Introduction}

In the medical care, operation in micro area is necessary. Therefore, many kinds of robots are researched ${ }^{(1)-(3)}$. In the almost robots, transmission of haptic sensation can not be achieved. However, in the medical robots, transmission of haptic sensation is desired. Bilateral control system is proposed as one of the master-slave teleoperation robots ${ }^{(4)(5)}$. It can transmit haptic sensation between master motor and slave motor. Many control systems based on bilateral control system are proposed. In this paper, the macro-micro bilateral control system ${ }^{(6)(7)}$ which is based on bilateral control system is focused. The macro-micro bilateral control system consists of macro master motor and micro slave motor. In this system, position and force of master motor are scaled down, and those of slave motor are enlarged. The macro-micro bilateral control system can transmit haptic sensation of micro environment. Therefore, this system is useful for the sensitive operation such as minimally invasive surgery.

When master motor mass and slave motor mass are different, in order to realize the transmission of vivid force sensation from the environment, standardization matrix is proposed ${ }^{(8)}$. By the standardization, the mass ratio of master and slave motors is compensated. The standardization is conducted mass ratio of master and slave motor. In order to achieve high accurate control, the scaling of control gains is proposed ${ }^{(9)}$. In this case, the position gains of master and slave motor are different. The position gains are decided by scaling gain ratio of position and force.

In the macro-micro bilateral control system, position and force of slave motor are enlarged. Therefore, disturbances of slave motor are also enlarged. In this paper, linear motors are used as master and slave motor. Dominant disturbances of linear motors are frictions. In general, frictions are modeled as kinematic friction and static friction. In order to simplify the analysis, in this paper, only kinematic friction is considered. The kinematic friction is modeled as viscous friction

\footnotetext{
* Department of System Design Engineering, Keio University

3-14-1, Hiyoshi, Kohoku-ku, Yokohama, Kanagawa, 223-8522 Japan
}

and Coulomb friction.

In this paper, effects of friction for macro-micro bilateral control system are considered based on reproducibility and operationality ${ }^{(10)}$. The effects of viscous friction and Coulomb friction are separately considered. The friction coefficients are identified by using macro-micro bilateral control system. If scaling gains of position and force are different, actual environmental impedance can not be transmitted from slave motor to master motor ${ }^{(11)}$. Because, in macromicro bilateral control system, environmental impedance is dependent on scaling gains of position and force. The viscous friction is also dependent on scaling gains of position and force. On the other hand, the Coulomb friction is dependent on scaling gain of force only. By changing the scaling gains, the effects of the viscous friction and Coulomb friction can be separately enlarged. The frictions are compensated by using identified friction coefficients in the macro-micro bilateral control system.

This paper is organized as follows; Section 2 shows the macro-micro bilateral control system. In Section 3, the reproducibility and operationality of macro-micro bilateral control system are explained. The effects of frictions are considered in Section 4. In Section 5, the identification of friction coefficients by macro-micro bilateral control system is explained. In Section 6, experiments are carried out. Finally, this paper is summarized in Section 7.

\section{Macro-Micro Bilateral Control}

In this section, macro-micro bilateral control is explained. The action force of human and the scaled reaction force satisfy the law of action and reaction. The scaled position of slave motor tracks to the position of master motor. The goals of macro-micro bilateral control are represented as follows;

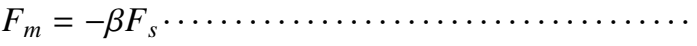

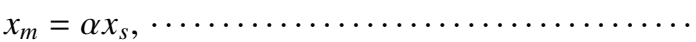

where $F, x, \alpha, \beta, \bigcirc_{m}$ and $\bigcirc_{s}$ denote force, position, scaling gain of position, scaling gain of force, master and slave, respectively. The block diagram of macro-micro bilateral control is shown in Fig. 1, where $C_{p}(s), C_{f}, \bigcirc^{r e f}, \bigcirc^{e x t}, \bigcirc^{r e s}$ and 


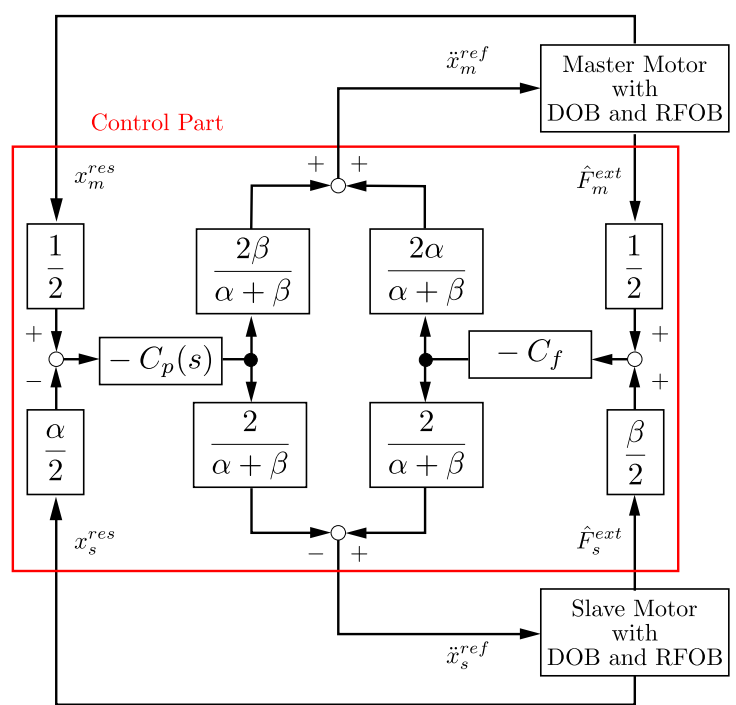

Fig. 1. Block diagram of macro-micro bilateral control system

$\hat{O}$ denote position regulator, force gain, reference, external, response and estimated value, respectively. The master motor and the slave motor have DOB (Disturbance Observer) and RFOB (Reaction Force Observer) ${ }^{(13)(14)}$. The position regulator is PD controller as follows;

$$
C_{p}(s)=K_{p}+K_{v} s
$$

where $K_{p}$ and $K_{v}$ denote position gain and velocity gain. In order to implement $K_{v} s$, pseudo differential is used.

\section{Reproducibility and Operationality}

In this section, reproducibility and operationality of macro-micro bilateral control system are explained. Reproducibility means reproductions accuracy of the environmental impedance. In an ideal macro-micro bilateral control system, operator can feel only environmental reaction force. However, in the actual macro-micro bilateral control system, operator feels not only environmental reaction force but also other force. The other force is operationality force. Therefore, operationality means the force which is in the master motor except the environmental reaction force. The relation between master and slave motor is shown with hybrid matrix as follows;

$$
\left[\begin{array}{l}
F_{m} \\
x_{m}
\end{array}\right]=\left[\begin{array}{ll}
H_{11} & H_{12} \\
H_{21} & H_{22}
\end{array}\right]\left[\begin{array}{c}
x_{s} \\
-F_{s}
\end{array}\right]
$$

The environment reaction force is shown as follows;

$$
F_{s}=-Z_{e} x_{s}
$$

where $Z_{e}$ denotes environmental impedance. The relation between master motor and environmental impedance is shown as follows;

$$
F_{m}=\left(\frac{H_{12}}{H_{21}+H_{22} Z_{e}} Z_{e}+\frac{H_{11}}{H_{21}+H_{22} Z_{e}}\right) x_{m} . \cdots \cdots
$$

The reproducibility and operationality of macro-micro bilateral control system are shown as follows;

$$
P_{r}=\frac{H_{12}}{H_{21}+H_{22} Z_{e}}
$$

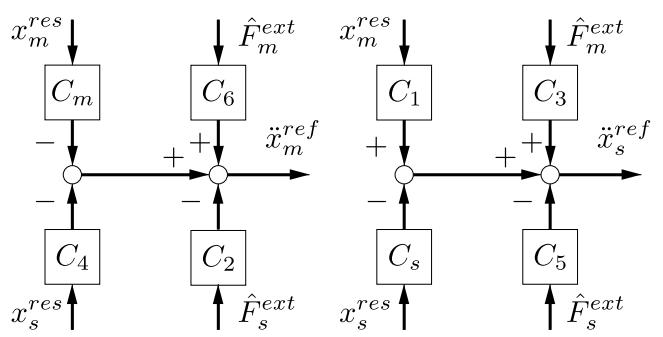

Fig. 2. Block diagram of control part

$$
P_{o}=\frac{H_{11}}{H_{21}+H_{22} Z_{e}},
$$

where $P_{r}$ and $P_{o}$ denote reproducibility and operationality. Fig. 2 shows control part block diagram of Fig. 1 as 4ch bilateral control system ${ }^{(15)}$, where $C$ denotes control gain. The parameters of hybrid matrix (4) are calculated from Fig. 2 and shown as follows;

$$
\begin{aligned}
& H_{11}=\frac{C_{1} C_{4}+\left(s^{2}+C_{m}\right)\left(s^{2}+C_{s}\right)}{C_{1} C_{6}+C_{3}\left(s^{2}+C_{m}\right)} \\
& H_{12}=-\frac{C_{1} C_{2}+C_{5}\left(s^{2}+C_{m}\right)}{C_{1} C_{6}+C_{3}\left(s^{2}+C_{m}\right)} \cdots \cdots \\
& H_{21}=\frac{-C_{3} C_{4}+C_{6}\left(s^{2}+C_{s}\right)}{C_{1} C_{6}+C_{3}\left(s^{2}+C_{m}\right)} \cdots \cdots \\
& H_{22}=\frac{C_{2} C_{3}-C_{5} C_{6}}{C_{1} C_{6}+C_{3}\left(s^{2}+C_{m}\right)} . \cdots \cdots
\end{aligned}
$$

The macro-micro bilateral control systems controller gains of (9) to (12) are calculated from Fig. 1 and Fig. 2. They are shown as follows;

$$
\begin{aligned}
& -\frac{\alpha+\beta}{\alpha \beta} C_{4}=\frac{\alpha+\beta}{\beta} C_{m}=C_{p}(s) \\
& (\alpha+\beta) C_{1}=\frac{\alpha+\beta}{\alpha} C_{s}=C_{p}(s) \\
& \frac{\alpha+\beta}{\alpha \beta} C_{2}=-\frac{\alpha+\beta}{\alpha} C_{6}=C_{f} \cdots \\
& -(\alpha+\beta) C_{3}=\frac{\alpha+\beta}{\beta} C_{5}=C_{f} .
\end{aligned}
$$

(13) to (16) are substituted in (9) to (12). The hybrid matrix is obtained as follows;

$$
\left[\begin{array}{ll}
H_{11} & H_{12} \\
H_{21} & H_{22}
\end{array}\right]=\left[\begin{array}{cc}
-\frac{\alpha+\beta}{C_{f}} s^{2} & \beta \\
\alpha & 0
\end{array}\right]
$$

The reproducibility and operationality of macro-micro bilateral control system are calculated as follows by (7), (8) and (17),

$$
\begin{aligned}
& P_{r}=\frac{H_{12}}{H_{21}+H_{22} Z_{e}}=\frac{\beta}{\alpha} \ldots \ldots \\
& P_{o}=\frac{H_{11}}{H_{21}+H_{22} Z_{e}}=-\frac{\alpha+\beta}{\alpha C_{f}} s^{2}
\end{aligned}
$$

The relation between master motor and environmental impedance of macro-micro bilateral control system is shown as follows;

$$
F_{m}=\frac{\beta}{\alpha} Z_{e} x_{m}-\frac{\alpha+\beta}{\alpha C_{f}} \ddot{x}_{m} .
$$


The environmental impedance is scaled depending on scaling gain $\alpha$ and $\beta$. In order to transmit the actual environmental impedance, the scaling gain of position and force must be equal. There is Operationality in (20). Therefore, the operator feels force except for reaction force. But if the force gain is getting larger, operationality becomes smaller.

\section{Analysis of Friction Effect}

In this section, the effects of frictions for macro-micro bilateral control system are considered. The friction of slave motor consists of viscous friction and Coulomb friction. The friction model of slave system is shown as follows;

$$
F_{\text {fric }}=-D_{\text {vis }} \dot{x}_{s}-F_{\text {coul }} \operatorname{sgn}\left(\dot{x}_{s}\right),
$$

where $F_{\text {fric }}, D_{\text {vis }}$ and $F_{\text {coul }}$ denote friction force, coefficient of viscous friction and force of Coulomb friction. The relation among slave motor, environmental impedance and friction is shown as follows;

$$
\begin{aligned}
F_{s} & =-Z_{e} x_{s}-D_{\text {vis }} \dot{x}_{s}-F_{\text {coul }} \operatorname{sgn}\left(\dot{x}_{s}\right) \\
& =-Z_{e}^{\prime} x_{s}-F_{\text {coul }} \operatorname{sgn}\left(\dot{x}_{s}\right), \cdots \cdots
\end{aligned}
$$

where $Z_{e}^{\prime}$ denotes combination value of environmental impedance and viscous friction.

$$
Z_{e}^{\prime}=Z_{e}+D_{v i s} s
$$

The reproducibility and operationality of macro-micro bilateral control system are calculated with (22). The reproducibility and operationality are shown as follows;

$$
F_{m}=\frac{\beta}{\alpha} Z_{e}^{\prime} x_{m}-\frac{\alpha+\beta}{\alpha C_{f}} \ddot{x}_{m}+\beta F_{\text {coul }} \operatorname{sgn}\left(\dot{x}_{s}\right) . \cdots \cdots
$$

The environmental impedance is scaled depending on scaling gain $\alpha$ and $\beta$. Therefore, if $\alpha: \beta$ is $1: 1$ to transmit actual impedance, the environmental impedance and viscous friction are not scaled. However, the Coulomb friction is scaled dependent on force scaling gain $\beta$. Therefore, even if $\alpha: \beta$ is $1: 1$, the Coulomb friction is scaled.

\section{Disturbance Identification}

In this section, separately identification method of viscous friction and Coulomb friction is explained. When free motion is carried out, the environmental impedance is zero. Therefore, in this case, the force of master motor is shown as follows;

$$
F_{m}=\frac{\beta}{\alpha} D_{\text {vis }} \dot{x}_{m}-\frac{\alpha+\beta}{\alpha C_{f}} \ddot{x}_{m}+\beta F_{\text {coul }} \operatorname{sgn}\left(\dot{x}_{s}\right) . \ldots \ldots
$$

In order to separate the effects of viscous friction and Coulomb friction, two free motions which have the same motions and different scaling gains $\alpha_{1}, \beta_{1}, \alpha_{2}$ and $\beta_{2}$ are carried out. In this case, the forces of master are shown as follows;

$$
\begin{aligned}
& F_{m 1}=\frac{\beta_{1}}{\alpha_{1}} D_{v i s} \dot{x}_{m}-\frac{\alpha_{1}+\beta_{1}}{\alpha_{1} C_{f}} \ddot{x}_{m}+\beta_{1} F_{\text {coul }} \operatorname{sgn}\left(\dot{x}_{s}\right) \cdots \\
& F_{m 2}=\frac{\beta_{2}}{\alpha_{2}} D_{v i s} \dot{x}_{m}-\frac{\alpha_{2}+\beta_{2}}{\alpha_{2} C_{f}} \ddot{x}_{m}+\beta_{2} F_{\text {coul }} \operatorname{sgn}\left(\dot{x}_{s}\right) \cdots
\end{aligned}
$$

When $\alpha_{1}: \beta_{1}$ and $\alpha_{2}: \beta_{2}$ are $1: 1, \beta_{1}$ and $\beta_{2}$ are not the same, the difference between $F_{m 1}$ and $F_{m 2}$ is shown as follows;

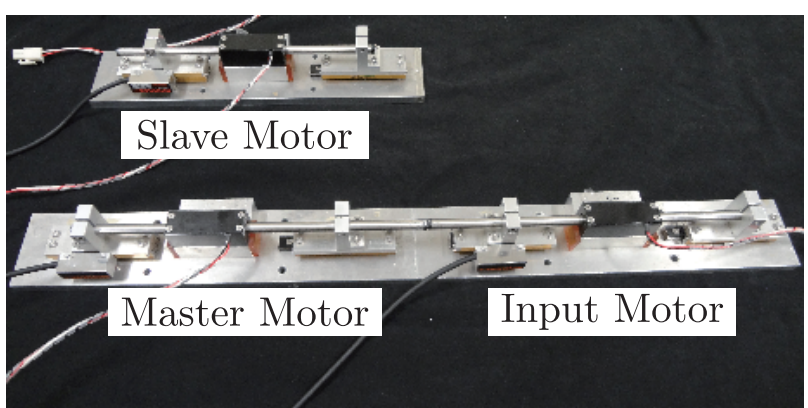

Fig. 3. Experimental setup

$$
F_{m 1}-F_{m 2}=\beta_{1} F_{\text {coul }} \operatorname{sgn}\left(\dot{x}_{s}\right)-\beta_{2} F_{\text {coul }} \operatorname{sgn}\left(\dot{x}_{s}\right) . \cdots
$$

(28) is an effect of Coulomb friction. When $\beta_{1}: \beta_{2}$ is $1: 1, \alpha_{1}$ and $\alpha_{2}$ are not the same, the difference between $F_{m 1}$ and $F_{m 2}$ is shown as follows;

$$
\begin{aligned}
F_{m 1}-F_{m 2}= & \left.\frac{\beta_{1}}{\alpha_{1}}-\frac{\beta_{2}}{\alpha_{2}}\right) D_{v i s} \dot{x}_{m} \\
& +\left(-\frac{\alpha_{1}+\beta_{1}}{\alpha_{1} C_{f}}+\frac{\alpha_{2}+\beta_{2}}{\alpha_{2} C_{f}}\right) \ddot{x}_{m} \cdots \cdots
\end{aligned}
$$

It is an effect of viscous friction and operationality. When the master motor moves with constant velocity, the operationality is zero. Therefore, (29) becomes the only effect of viscous friction. The independent identification of viscous friction and Coulomb friction are achieved. In this method, the viscous friction and Coulomb friction can be separated. These effects can be enlarged. Therefore, the identification can be carried out easily.

\section{Experiments}

In this section, the experiments of identification and compensation are carried out. Firstly, the identification of frictions is carried out. Accordingly, the compensation of friction is carried out. Experimental setup is shown in Fig. 3. It consists of master motor, slave motor and input motor. The master motor and the slave motor are satisfied with the macromicro bilateral control system.

In the identification experiments, master motor must be moved with constant velocity. Therefore, the input motor manipulates the master motor with constant velocity.

In the friction compensation experiments, the accuracy of macro-micro bilateral control system should be obtained. Therefore, in order to implement fair manipulations of master motor, the input motor manipulates the master motor instead of human.

6.1 Identification of Coulomb Friction In this section, the identifications of Coulomb friction are carried out.

It is defined that the Coulomb friction when the velocity of motor is plus and the Coulomb friction when velocity of motor is negative are different.

The input motor moves with constant velocity $0.015 \mathrm{~m} / \mathrm{s}$. Twice free motions are carried out. The scaling gains are set to difference values as follows;

$$
\begin{aligned}
& \alpha_{1}=10, \quad \beta_{1}=10 \ldots \ldots \ldots \ldots \ldots \ldots \ldots \ldots \ldots \\
& \alpha_{2}=1, \quad \beta_{2}=1 \text {. }
\end{aligned}
$$

Results of free motions are shown in Fig. 4. The upper graph 

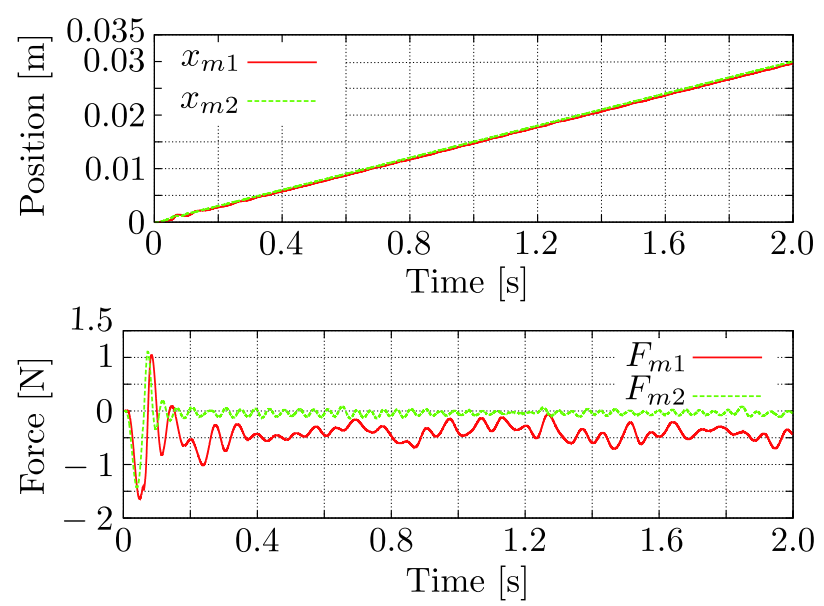

Fig. 4. Experimental results when velocity is plus with $\alpha_{1}=10, \beta_{1}=10, \alpha_{2}=1$ and $\beta_{2}=1$

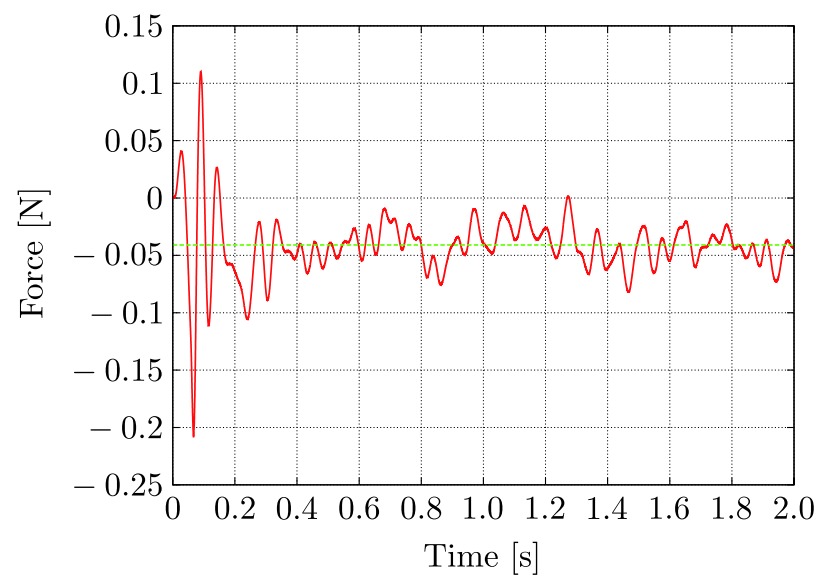

Fig. 5. Identification of Coulomb friction when velocity is plus

shows that the two results have the same velocity. The difference between $F_{m 1}$ and $F_{m 2}$ due to scaling gain is shown as follows according to (28);

$$
F_{m 1}-F_{m 2}=9 F_{\text {coul }} \operatorname{sgn}\left(\dot{x}_{s}\right) .
$$

The value of Coulomb friction can be calculated by (33)

$$
F_{\text {coul }} \operatorname{sgn}\left(\dot{x}_{s}\right)=\frac{F_{m 1}-F_{m 2}}{9} .
$$

The value of (33) is shown as red line in Fig. 5. The result is not constant value, because the master motor has linear slider and linear slider is inequable. Therefore, the frictions are functions of motor position. However, the constant Coulomb friction and viscous friction are wanted to compensation of frictions. Furthermore, in the result, there is static friction. Therefore, the averages of friction results are used as identified friction values. If average is used, the effect of motor position and static friction become little.

The average of (33) is $-0.041 \mathrm{~N}$ and it is shown as green line in Fig. 5. This value is used as the Coulomb friction when velocity is plus.

Next, the Coulomb friction is identified under the condition that velocity of motor is negative. The input motor moves with constant velocity $-0.015 \mathrm{~m} / \mathrm{s}$. The scaling gains are the same with (30) and (31). Results of free motions are shown
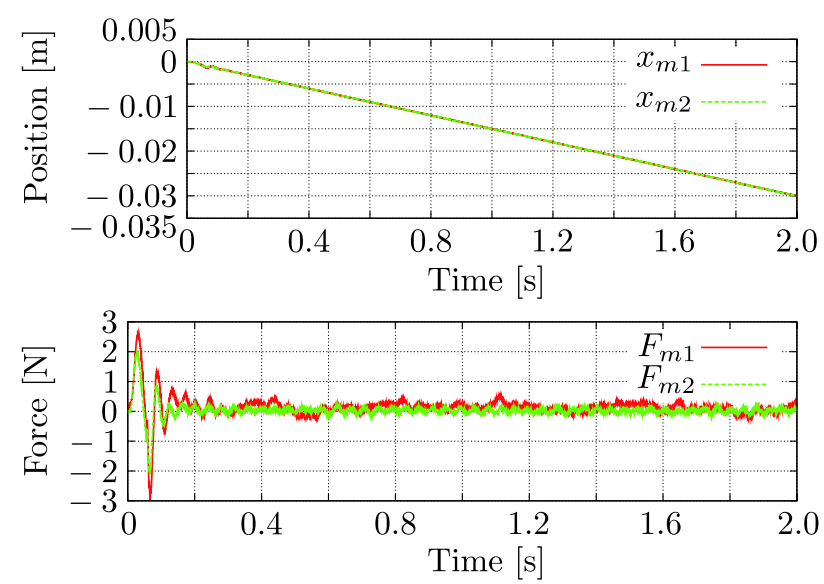

Fig. 6. Experimental results when velocity is negative with $\alpha_{1}=10, \beta_{1}=10, \alpha_{2}=1$ and $\beta_{2}=1$

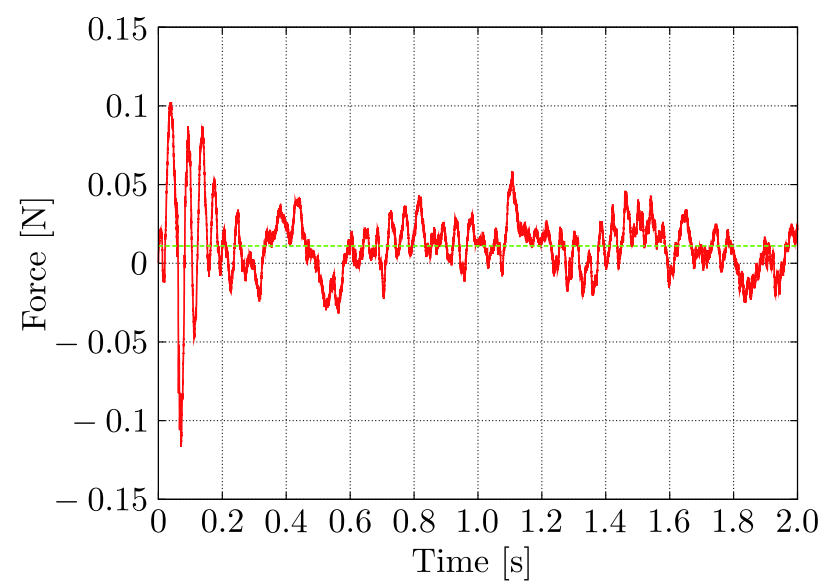

Fig. 7. Identification of Coulomb friction when velocity is negative

in Fig. 6. At this time, the value of (33) is shown as red line in Fig. 7. The average of (33) is $0.011 \mathrm{~N}$ and it is shown as green line in Fig. 7. This value is used as the Coulomb friction when velocity is negative.

6.2 Identification of Viscous Friction In this part, the viscous friction is identified. The scaling gains of free motions are set as follows;

$$
\begin{aligned}
& \alpha_{1}=1, \quad \beta_{1}=10 . \\
& \alpha_{2}=10, \quad \beta_{2}=10 .
\end{aligned}
$$

The velocity of input motor is set to $0.015 \mathrm{~m} / \mathrm{s}$. Results of free motions are shown in Fig. 8. The velocity of master motor is constant and acceleration is zero. Therefore, operationality is zero. The difference between $F_{m 1}$ and $F_{m 2}$ is calculated as follows according to (29)

$$
F_{m 1}-F_{m 2}=9 D_{v i s} \dot{x}_{m}
$$

The value of viscous friction can be calculated by (36). The velocity of master motor is $0.015 \mathrm{~m} / \mathrm{s}$

$$
D_{v i s}=\frac{F_{m 1}-F_{m 2}}{9 \dot{x}_{m}} \text {. }
$$

The value of (37) is shown in Fig. 9. The average of (37) is -0.012 and it is shown as green line in Fig. 9. This value is used as viscous friction when velocity of motor is plus. 

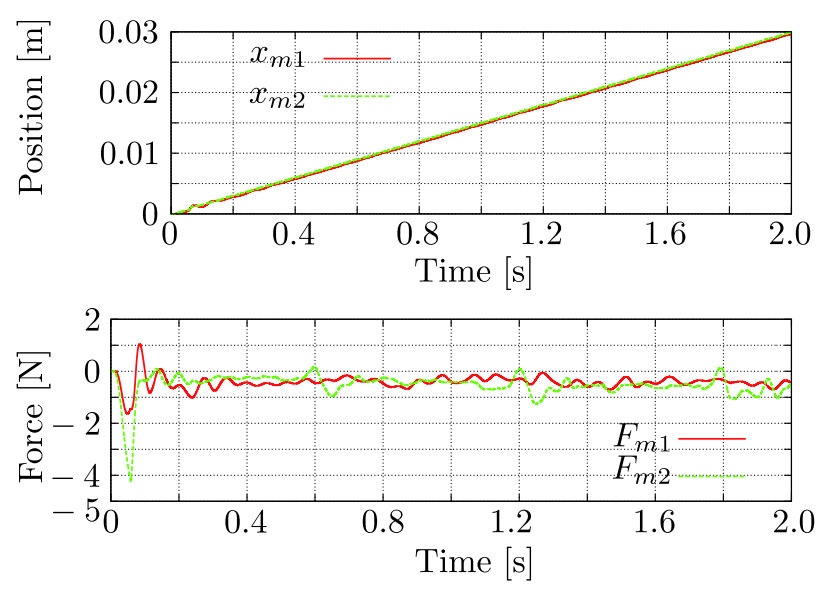

Fig. 8. Experimental results when velocity is plus with $\alpha_{1}=1, \beta_{1}=10, \alpha_{2}=10$ and $\beta_{2}=10$

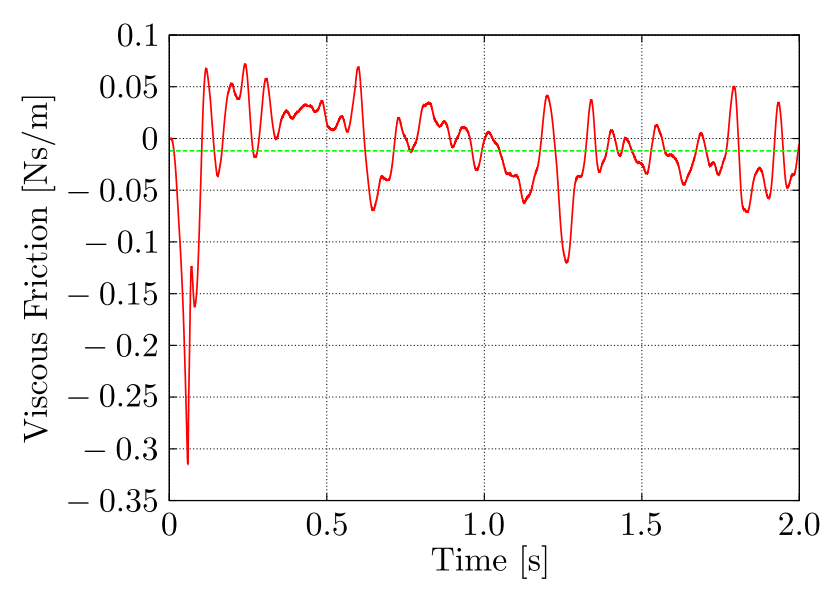

Fig. 9. Identification of viscous friction when velocity is plus
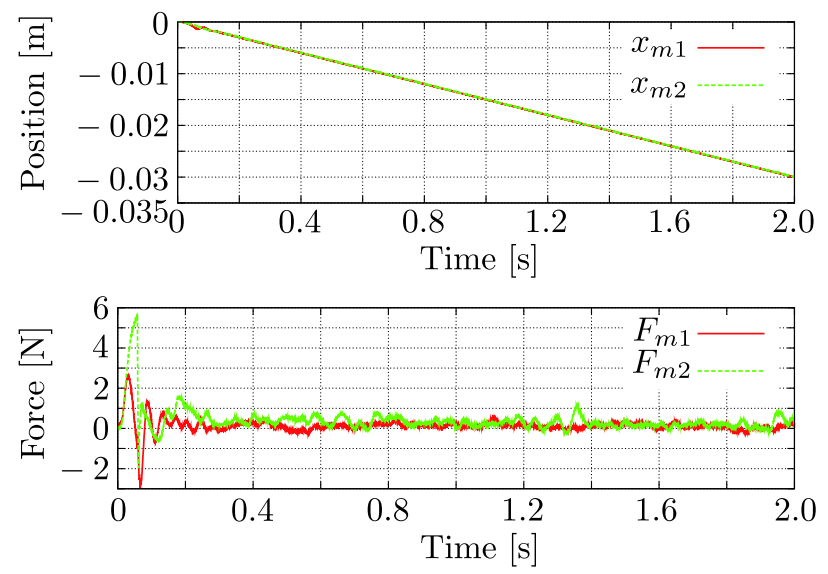

Fig. 10. Experimental results when velocity is negative with $\alpha_{1}=1, \beta_{1}=10, \alpha_{2}=10$ and $\beta_{2}=10$

Next, the viscous friction is identified under the condition that velocity of motor is negative. The velocity of master motor is $-0.015 \mathrm{~m} / \mathrm{s}$. The results of free motions are shown in Fig. 10. The value of calculated viscous friction is shown in Fig. 11. The average of (37) is 1.89 and it is shown as green line in Fig. 11. This value is used as viscous friction when velocity of motor is negative.

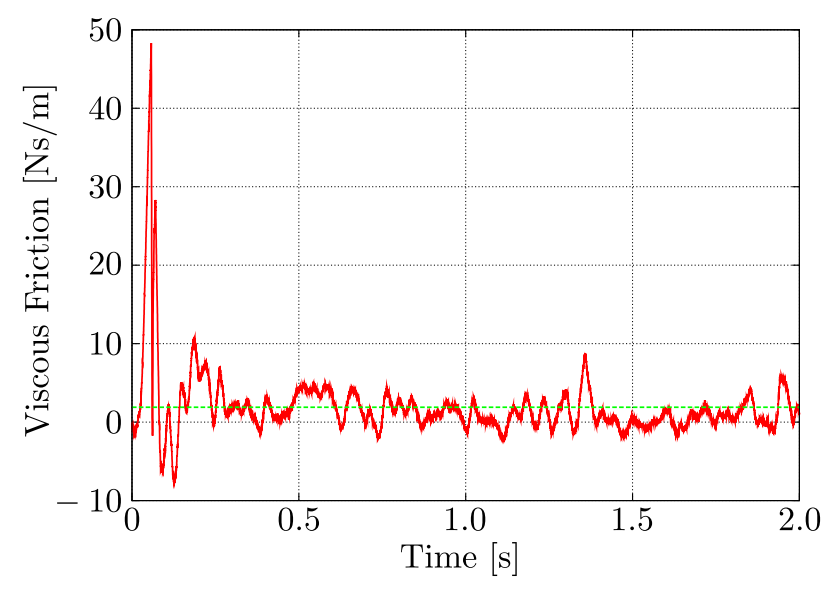

Fig. 11. Identification of viscous friction when velocity is negative
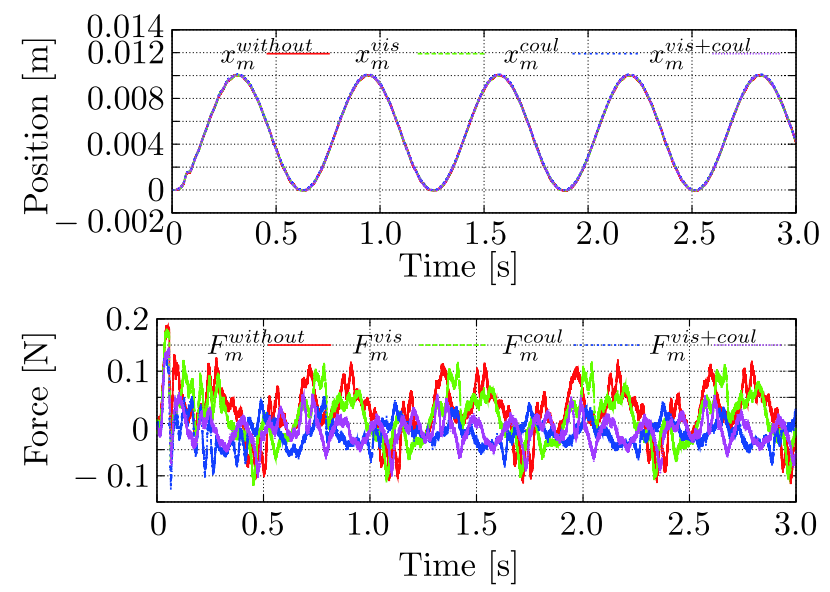

Fig. 12. Experimental results of compensation $(\alpha=10$, $\beta=10)$

6.3 Compensation of Friction In the previous parts, the coefficients of frictions are identified. In this part, the frictions are compensated according to the identified coefficients. The free motions with compensation are carried out with scaling gain $\alpha=10, \beta=10$ and $\alpha=20, \beta=20$. In order to transmit accurate environmental impedance, $\alpha$ and $\beta$ are the same. Free motions without compensation and with compensation are carried out. In order to compare, compensation of viscous friction, compensation of Coulomb friction and compensation of viscous and Coulomb friction are carried out. The position command of input motor is set to $0.005(1-\cos (10 t))$. The results are shown in Figs. 12 and 13. $\bigcirc^{\text {without }}, \bigcirc^{\text {vis }}, \bigcirc^{\text {coul }}$ and $\bigcirc^{\text {vis+coul }}$ denote without compensation, compensation of only viscous friction, compensation of only Coulomb friction and compensation of viscous and Coulomb friction. In the ideal free motion, the force of master motor is zero. The force without compensation and the force with compensation of viscous friction are similar. The force with compensation of Coulomb friction and the force with compensation of viscous and Coulomb friction are similar, because the effect of viscous friction is not enlarged. On the other hand, the effect of Coulomb friction is enlarged. Therefore, the compensation of Coulomb friction is improves the performance of macro-micro bilateral control system. 

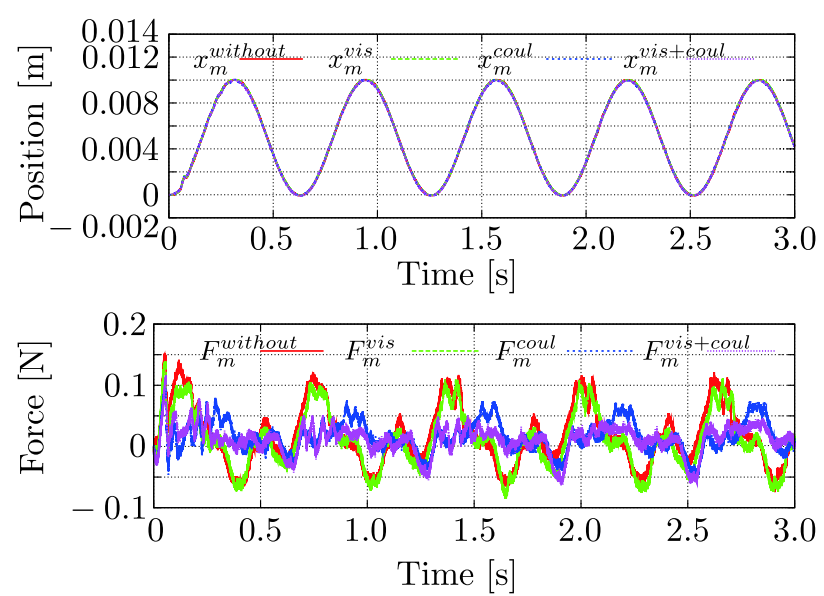

Fig. 13. Experimental results of compensation $(\alpha=20$, $\beta=20$ )

\section{Conclusion}

In this paper, effects of frictions for macro-micro bilateral control system were considered with reproducibility and operationality. The frictions consisted of viscous friction and Coulomb friction. The viscous friction was not enlarged if scaling gain of position and force was the same. On the other hand, Coulomb friction was enlarged according to scaling gain of force. The friction coefficient was identified by using macro-micro bilateral control system. By using macro-micro bilateral control system, the viscous friction and Coulomb friction can be separated. In order to identify the friction coefficient, the effect of friction can be enlarged by changing the scaling gain.

\section{Acknowledgments}

This research was partially supported by the Ministry of Education, Culture, Sports, Science and Technology, Grantin-Aid for Scientific Research for Young Scientists (A), 24686037, 2012.

\section{References}

( 1 ) W. Mcmahan, J. Gewirtz, D. Standish, P. Martin, J.A. Kunkel, M. Lilavois, A. Wedmid, D.I. Lee, and K.J. Kuchenbecker: "Tool Contact Acceleration Feedback for Telerobotic Surgery", IEEE Trans. on Haptics, Vol.4, No.3, pp.210-220 (2011)

( 2 ) H. Ishihara, F. Arai, and T. Fukuda: "Micro Mechatronics and Micro Actuators”, IEEE/ASME Trans. on Mechatronics, Vol.1, No.1, pp.68-79 (1996)

( 3 ) K. Ikuta, Y. Matsuda, D. Yajima, and Y. Ota: "Pressure Pulse Drive: A Control Method for the Precise Bending of Hydraulic Active Catheters", IEEE/ASME Trans. on Mechatronics, Vol.17, No.5, pp.876-883 (2012)

( 4 ) K. Hashtrudi-Zaad and S.E. Salcudean: "On the Use of Local Force Feedback for Transparent Teleoperation", Proc. of the 1999 International Conference on Robotics and Automation, pp.1863-1869 (1999)

( 5 ) K. Hashtrudi-Zaad and S.E. Salcudean: "Bilateral Parallel Force/Position Teleoperation Control”, Journal of Robotic Systems, Vol.19, No.4, pp.155167 (2002)

(6) A. Sano, H. Fujimoto, and T. Takai: "Personal Scaling on MicroTeleoperation", 26th Annual Conference of the IEEE Industrial Electronics Society, IECON'00, pp.7-12 (2000)
( 7 ) J.E. Speich and M. Goldfarb: "An Implementation of Loop-Shaping Compensation for Multidegree-of-Freedom Macro-Microscaled Telemanipulation”, IEEE Trans. on Control Systems Technology, Vol.13, No.3, pp.459-464 (2005)

( 8 ) T. Shimono, S. Katsura, S. Susa, T. Takei, and K. Ohnishi: "Transmission of Real World Force Sensation by Micro/Macro Bilateral Control Based on Acceleration Control with Standardization Matrix", IEEJ Trans. IA, Vol.128-D, No.6, pp.726-732 (2008)

( 9 ) S. Susa, T. Shimono, and K. Ohnishi: "Micro-Macro Bilateral Control Taking into Account Scaling of Control Gains", IEEJ Trans. IA, Vol.129-D, No.2, pp.150-157 (2009)

(10) S. Susa, T. Shimono, T. Takei, K. Atsuta, N. Shimojima, S. Ozawa, Y. Morikawa, and K. Ohnishi: "Transmission of Force Sensation by MicroMacro Bilateral Control with Scaling of Control Gains", The 10th IEEE International Workshop on Advanced Motion Control, AMC'08, pp.532-537 (2008)

(11) K. Kaneko, H. Tokashiki, K. Tanie, and K. Komoriya: "Impedance Shaping based on Force Feedback Bilateral Control in Macro-Micro Teleoperation System", Proc. of the IEEE International Conference on Robotics and Automation, ICRA'97, pp.710-717 (1997)

(12) S. Katsura and K. Ohnishi: "Quarry of Modal Information from Environment for Advanced Motion Control”, IEEJ Trans. IA, Vol.126, No.4, pp.372-378 (2006)

(13) K. Ohnishi, M. Shibata, and T. Murakami: "Motion Control for Advanced Mechatronics", IEEE/ASME Trans. on Mechatronics, Vol.1, No.1, pp.56-67 (1996)

(14) T. Murakami, F. Yu, and K. Ohnishi: "Torque Sensorless Control in Multidegree-of-freedom Manipulator", IEEE Trans. Industrial Electronics, Vol.40, No.2, pp.259-265 (1993)

(15) W. Iida and K. Ohnishi: "Reproducibility and Operationality in Bilateral Teleoperation", The 8th IEEE International Workshop on Advanced Motion Control, AMC'04, pp.217-222 (2004)

Yosuke Mizutani (Student Member) received his B.E. degree in sys-

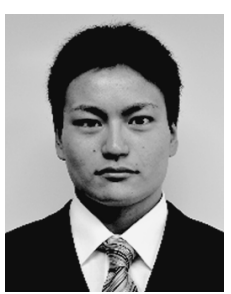
tem design engineering from Keio University, Yokohama, Japan, in 2012. Since 2012, he has been Master's course at Keio University, Yokohama, Japan. His research interests include real-world haptics and motion control. He is a Student Member of IEEJ, as well as IEEE.

Seiichiro Katsura (Senior Member) received his B.E. degree in sys-

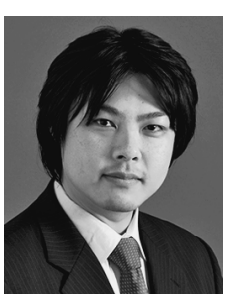
tem design engineering and his M.E. and Ph.D. degrees in integrated design engineering from Keio University, Yokohama, Japan, in 2001, 2002 and 2004, respectively. From 2003 to 2005, he was a Research Fellow of the Japan Society for the Promotion of Science. From 2005 to 2008, he worked at Nagaoka University of Technology, Nagaoka, Niigata, Japan. Since 2008, he has been at Keio University, Yokohama, Japan. His research interests include realworld haptics, human support space, systems energy conversion, and electromechanical integration systems. Prof. Katsura received the Best Paper Award from the Institute of Electrical Engineers of Japan (IEEJ) in 2003, the Dr. Yasujiro Niwa Outstanding Paper Award in 2004, The European Power Electronics and Drives-Power Electronics and Motion Control Conference, EPE-PEMC'08 Best Paper Award in 2008, and the 4th IEEE International Conference on Human System Interaction, HSI'11 Best Paper Award in 2011. He is a Senior Member of IEEJ, as well as a Member of the IEEE, EPE, The Society of Instrument and Control Engineers (SICE), The Japan Society of Mechanical Engineers (JSME), The Japan Society for Precision Engineering (JSPE), Robotics Society of Japan (RSJ), The Institute of Electronics, Information and Communication Engineers (IEICE), and The Japan Society of Computer Aided Surgery (JSCAS). 